\title{
Some Hamiltonian Results in Powers of Graphs*
}

\author{
Arthur M. Hobbs**
}

(November 16, 1972)

\begin{abstract}
In this paper we show that the connectivity of the $k$ th power of a graph of connectivity $m$ is at least $\mathrm{km}$ if the $k$ th power of the graph is not a complete graph. Also, we, prove that removing as many as $k-2$ vertices from the $k$ th power of a graph $(k \geqslant 3)$ leaves a Hamiltonian graph, and that removing as many as $k-3$ vertices from the $k$ th power of a graph $(k \geqslant 3)$ leaves a Hamiltonian connected graph. Further, if every vertex of a graph has degree two or more, then the square of the graph contains a 2 -factor. Finally, we show that the squares of certain Euler graphs are Hamiltonian.
\end{abstract}

Key words: Combinatorics; connectivity; Euler graphs; 2 -factors; graph theory; Hamiltonian circuits; Hamiltonian connected; powers of graphs.

\section{Introduction}

We use the notation and terminology of $[11]^{1}$, with the terms "point", "line", and "cycle" replaced by vertex, edge, and circuit. Further, we denote the set of edges of a graph $G$ by $E(G)$. We follow the practice of representing a path by the sequence of vertices of the path. To distinguish between a path (circuit) $p$ and the graph whose vertices and edges are exactly those of $p$, we denote the graph by $/ p /$, and we call the graph a pathoid (circuitoid). We denote the distance between two vertices $\alpha$ and $\beta$ in a graph $G$ by $d_{G}(\alpha, \beta)$, and we denote the degree of a vertex $\alpha$ in $G$ by $v_{G}(\alpha)$. The undirected edge joining vertices $\alpha$ and $\beta$ is denoted by $(\alpha, \beta)$ or $(\beta, \alpha)$ interchangeably.

Given an integer $k \geqslant 1$, the $k$ th power $G^{k}$ of a graph $G$ is a graph with $V\left(G^{k}\right)=V(G)$ and $(\alpha, \beta) \epsilon E\left(G^{k}\right)$ iff $d_{G}(\alpha, \beta) \epsilon\{1,2, \ldots, k\} . G^{2}$ is called the square of $G$, and $G^{3}$ is called the cube of $G$. Given a path $p=\alpha_{0}, \alpha_{1}, \ldots ., \alpha_{k}$, we let $F(p)=\alpha_{0}, L(p)=\alpha_{k}, V(p)=\left\{\alpha_{0}, \alpha_{1}, \ldots ., \alpha_{k}\right\}$, and $I(p)=V(p)-\{F(p), L(p)\}$. We call $p$ an $\left[\alpha_{0}, \alpha_{k}\right]$-path iff $F(p)=\alpha_{0}$ and $L(p)=\alpha_{k}$. Paths $p$ and $q$ are internally disjoint iff $I(p) \cap I(q)$ is empty. The number of elements in a set $S$ is denoted by $|S|$.

\section{Connectivity}

Our first two theorems give useful information about a property of raising a graph to a $k$ th power and the structure of a graph once the operation has been carried out. The first theorem is an easy consequence of the definition of power of a graph.

Theorem 1: Let $\mathrm{G}$ be a graph and let $\mathrm{k}=\mathrm{mn}$, where $\mathrm{m}$ and $\mathrm{n}$ are both positive integers. Then $\mathrm{G}^{\mathrm{k}}=\left(\mathrm{G}^{\mathrm{m}}\right)^{\mathrm{n}}$.

We will use this theorem to show that the next theorem is best possible. The connectivity $\kappa(G)$ of a graph $G$ is the minimum over all pairs $\alpha, \beta$ of distinct vertices in $G$ of the maximum number of distinct internally disjoint $[\alpha, \beta]$-paths in $G$. Since raising a graph $G$ to the power $k$ usually increases the number of edges present, it is not unreasonable to conjecture that $G^{k}$ has higher connectivity than $G$ has. Thus,

AMS Subject Classification: 0540

*An invited paper. Partial support for this research has come to me through Professor W. T. Tutte from the National Research Council of Canada.

** Present address: Texas A and M University, College of Science, Department of Mathematics, College Station, Texas 77843.

${ }^{1}$ Figures in brackets indicate the literature references at the end of this paper. 
THEOREM 2: Let $\mathrm{G}$ be a finite graph, and let $\mathrm{k}$ be a positive integer. Then

$$
\kappa\left(G^{k}\right) \geqslant \min (|V(G)|-1, k \kappa(G)) .
$$

Proof: If $\kappa(G)=0$ or $k=1$, the theorem is immediate. Similarly, if $G^{k}$ is complete, the theorem holds because in that case, $\kappa\left(G^{k}\right)=|V(G)|-1$.

Now suppose $G^{k}$ is not complete, $\kappa(G)>0$, and $k>1$. Let $\xi$ and $\eta$ be vertices of $G$ which are not adjacent in $G^{k}$. Then by the definition of $G^{k}, d_{G}(\xi, \eta)>k$. Let $a_{1}, a_{2}, \ldots, a_{\kappa(G)}$ be $\kappa(G)$ internally disjoint $[\xi, \eta]$-paths in $G$, with $a_{i}=\xi, \gamma_{i, 1}, \gamma_{i, 2}, \ldots, \gamma_{i, r_{i}}, \eta$. Since $d_{G}(\xi, \eta)>k, r_{i} \geqslant k$ whenever $i \epsilon\{1,2, \ldots, \kappa(G)\}$. Suppose $r_{i}=k t_{i}+s_{i}$, with $0 \leqslant s_{i} \leqslant k-1$. Then in $G^{k}, \xi$ and $\eta$ are joined by the paths

$$
\begin{gathered}
\xi, \gamma_{i, 1}, \gamma_{i, k+1}, \gamma_{i, 2 k+1}, \ldots, \gamma_{i, t_{i} k+1}, \eta=p_{i, 1}, \\
\xi, \gamma_{i, 2}, \gamma_{i, k+2}, \gamma_{i, 2 k+2}, \ldots, \gamma_{i, t_{i} k+2}, \eta=p_{i, 2}, \\
\ldots \\
\xi, \gamma_{i, s_{i}}, \gamma_{i, k+s_{i}}, \gamma_{i, 2 k+s_{i}}, \ldots, \gamma_{i, t_{i} k+s_{i}}, \eta=p_{i, s_{i}}, \\
\xi, \gamma_{i, s_{i}+1}, \gamma_{i, k+s_{i}+1}, \gamma_{i, 2 k+s_{i}+1}, \ldots, \gamma_{i,\left(t_{i}-1\right) k+s_{i}+1}, \eta=p_{i, s_{i}+1}, \\
\xi \gamma_{i, s_{i}+2}, \gamma_{i, k+s_{i}+2}, \gamma_{i, 2 k+s_{i}+2}, \ldots, \gamma_{i,\left(t_{i}-1\right) k+s_{i}+2}, \eta=p_{i, s_{i}+2}, \\
\ldots \\
\xi, \gamma_{i, k}, \gamma_{i, 2 k}, \ldots, \gamma_{i,\left(t_{i}-1\right) k}, \eta=p_{k} .
\end{gathered}
$$

Each of these $k$ paths contains only vertices of $a_{i}$ and each path $a_{i}$ contains vertices which can be used to form $k$ internally disjoint $[\xi, \eta]$-paths in $G^{k}$ in this way. Since the paths $a_{1}, \ldots, a_{\kappa(G)}$ are internally disjoint, between $\xi$ and $\eta$ in $G^{k}$ there are $k \kappa(G)$ internally disjoint $[\xi, \eta]$-paths. Thus, $G^{k}$ is $k \kappa(G)$-connected.

COROLlary 2A: If $\mathrm{G}$ is a finite graph with connectivity $\kappa(\mathrm{G}) \geqslant 1$, if $\mathrm{k}$ is a positive integer, and if $\mathrm{k} \kappa(\mathrm{G}) \geqslant|\mathrm{V}(\mathrm{G})|-1$, then $\mathrm{G}^{\mathrm{k}}$ is a complete graph.

Theorem 2 is best possible in the sense that additional conditions are needed to improve it, as the following examples show. If $P$ is a pathoid with $n$ vertices, if $\xi \in V(P)$ and has degree 1 , and if $r$ is a positive integer less than $n$, then $\xi$ is of degree $r$ in $P^{r}$. Thus, by Theorem $2, P^{r}$ has connectivity exactly $r$. Given positive integers $k$ and $m$, and given $n \geqslant k m+1$, let $P$ be a pathoid of length $n-1$. Then $\left(P^{m}\right)^{k}=P^{m k}$ by Theorem 1 , and so it is a graph of connectivity $k m$ which is the $k$ th power of an $m$-connected graph. Finally, let $C_{n}$ be a circuitoid with $n$ vertices. Let $m>0$ and even, and let $k$ be a positive integer and $n$ be an integer no less than $k m+1$; then $C_{n}^{(k m) / 2}$ $=\left(C_{n}^{m / 2}\right)^{k}$ and is a minimally $k m$-connected graph which is the $k$ th power of a minimally $m$-connected graph.

\section{3. $r$-Hamiltonian Powers of Graphs}

Following [4] and [17], we call a graph $G$ Hamiltonian connected iff every two distinct vertices of $G$ are joined by a Hamiltonian path in $G$, and we call $G r$-Hamiltonian iff $|V(G)| \geqslant r+3$ and $G-\left\{\xi_{1}, \ldots, \xi_{r}\right\}$ is Hamiltonian for every set of $r$ vertices $\left\{\xi_{1}, \ldots, \xi_{r}\right\} \subseteq V(G)$. Given a path $p$, we let $l(p)$ denote the length of $p$. Given disjoint paths $p=\xi_{1}, \xi_{2}, \ldots, \xi_{r}$ and $q=\eta_{1}, \eta_{2}, \ldots, \eta_{s}$, if $\xi_{r}$ is adjacent in $G$ to $\eta_{1}$, we denote the path $\xi_{1}, \ldots, \xi_{r}, \eta_{1}, \eta_{2}, \ldots, \eta_{s}$ by $(p),(q)$. Let $\xi$ be a cut 
vertex of a connected graph $G$, and let $H_{1}, \ldots, H_{r}$ be all of the components of $G-\xi$. Then for each $j \epsilon\{1, \ldots, r\}$, we call the subgraph

$$
G-\bigcup_{\substack{i=1 \\ i \neq j}}^{r} V\left(H_{i}\right)
$$

of $G$ a $\xi$-section of $G$.

ConJecture 1: If $\mathrm{G}$ is a finite connected graph, $\mathrm{k}$ is an integer no less than 3 , and $\mathrm{A} \subseteq \mathrm{V}(\mathrm{G})$ such that $|\mathrm{A}| \leqslant \mathrm{k} \kappa(\mathrm{G})-3$, then $\mathrm{G}^{\mathrm{k}}-\mathrm{A}$ is Hamiltonian connected.

Conjecture 2: If $\mathrm{G}$ is a finite connected graph, $\mathrm{k}$ is an integer no less than 3 , and $\mathrm{r} \leqslant \min$ $(|\mathrm{V}(\mathrm{G})|-3, \mathrm{k} \kappa(\mathrm{G})-2)$, then $\mathrm{G}^{\mathrm{k}}$ is $\mathrm{r}$-Hamiltonian.

Because Theorem 2 is best possible and at least 2 -connectedness is require $\mathrm{d}$ for the presence of a Hamiltonian circuit in a graph, Conjecture 2 is best possible if it is true. Furthermore, if $0 \leqslant|V(G)|-3 \leqslant k \kappa(G)-2$, then $|V(G)|-1 \leqslant k \kappa(G)$ and $G^{k}$ is complete by Corollary $2 \mathrm{~A}$. In such a case, for any set $A \subseteq V(G)$ with $|A| \leqslant|V(G)|-3, G^{k}-A$ is Hamiltonian. Thus we need only consider Conjecture 2 for the case in which $|V(G)|-3 \geqslant k \kappa(G)-2$ and $|A| \leqslant k \kappa(G)-2$. We have not yet proven these conjectures in general, but in the next portion of this paper we give proofs for the case of $\kappa(G)=1$.

Let $G$ be a graph, $k$ a positive integer, and $A$ a subset of the vertices of $G$. Two distinct vertices $\xi$ and $\eta$ of $G$ are $A$-joined iff there exists a path $p$ in $G$ joining $\xi$ and $\eta$ such that $I(p) \subseteq A$. Note that two adjacent vertices are $A$-joined for every set $A$ of vertices in $G$. A path $p$ of $G$ is $(A, k)$-solid iff $\{F(p), L(p)\} \subseteq V(G)-A,|I(p)-A| \leqslant 2$, and $l(p)>k$.

LemmA 1: Let $\mathrm{k} \geqslant 3$ be an integer, $\mathrm{G}$ a finite graph, and $\mathrm{A} \subseteq \mathrm{V}(\mathrm{G})$ with $|\mathrm{A}| \leqslant \mathrm{k}-3$. Then $\mathrm{G}$ contains no (A, k)-solid paths.

Proof: This lemma is immediate from the definition of $(A, k)$-solid paths.

LeMMA 2: Let $\mathrm{k} \geqslant 3$ be an integer, let $\mathrm{G}$ be a graph, and let $\mathrm{A} \subseteq \mathrm{V}(\mathrm{G})$ such that $|\mathrm{A}| \leqslant \mathrm{k}-2$. If $\mathrm{a}$ is a path of $\mathrm{G}$ such that $|\mathrm{I}(\mathrm{a})-\mathrm{A}| \leqslant 1$, then $l(\mathrm{a}) \leqslant \mathrm{k}$.

Proof: Since $|A| \leqslant k-2$ and $|I(a)-A| \leqslant 1,|I(a)| \leqslant k-1$. But $l(a)=|I(a)|+1 \leqslant k-$ $1+1=k$.

We next prove a very strong result that holds for special subsets $A$ of vertices of a tree. Let $\phi$ be the empty set.

LeMmA 3: Let $\mathrm{k} \geqslant 3$ be an integer, let $\mathrm{T}$ be a finite tree, and let $\mathrm{A} \subseteq \mathrm{V}(\mathrm{T})$. Suppose no path in $\mathrm{T}$ is (A, $\mathrm{k})$-solid. If $\xi$ and $\eta$ are distinct $\mathrm{A}$-joined vertices in $\mathrm{V}(\mathrm{T})-\overline{\mathrm{A}}$, then $\mathrm{T}^{\mathrm{k}}-\mathrm{A}$ contains a Hamiltonian $[\xi, \eta]$-path.

Proof: If $|V(T)| \leqslant k+1$, then $T^{k}$ is complete by Corollary 2A. Hence $T^{k}-A$ is a complete graph with at least the two vertices $\xi$ and $\eta$, and so there is a Hamiltonian $[\xi, \eta]$-path in $T^{k}-A$.

Suppose the lemma is true for all trees $T^{\prime}$ with $\left|V\left(T^{\prime}\right)\right|<q$, and let $T$ be a tree with $q$ vertices which satisfies the conditions of the lemma. Let $T_{1}, T_{2}, \ldots, T_{r}, r \geqslant 1$, be the $\xi$-sections of $T$ for which $V\left(T_{i}\right)-A \neq \phi$, and suppose $\eta \in V\left(T_{r}\right)$. Since a path which is $(A, k)$-solid in $T_{i}, 1 \leqslant i \leqslant r$, is $(A, k)$-solid in $T$, the lemma holds for all subtrees $T_{i}$ which have at least 2 vertices not in $A$. In tree $T_{i}, 1 \leqslant i \leqslant r-1$, let $\gamma_{i, 1}$ be a vertex of $V\left(T_{i}\right)-A$ which is $A$-joined to $\xi$ in $T$, and let $\gamma_{r, 1}=\eta$, which is $A$-joined to $\xi$ by assumption. In tree $T_{i}, 1 \leqslant i \leqslant r$, if $V\left(T_{i}\right)-\left(A \cup\left\{\gamma_{i, 1}\right\}\right) \neq \phi$, let $\gamma_{i, 2}$ be a vertex of $V\left(T_{i}\right)-\left(A \cup\left\{\gamma_{i, 1}\right\}\right)$ which is $A$-joined to $\gamma_{i, 1}$ in $T$, and if $V\left(T_{i}\right)-(A \cup$ $\left.\left\{\gamma_{i, 1}\right\}\right)=\phi$, let $\gamma_{i, 2}=\gamma_{i, 1}$.

Since $T$ is a tree, there is a unique $\left[\xi, \gamma_{1,2}\right]$-path $a$ in $T$, and $\gamma_{1,1}$ is the only vertex in $I(a)-A$; by the definition of $(A, k)$-solid path, $\ell(a) \leqslant k$ since $a$ is not $(A, k)$-solid. Thus $d_{T}\left(\xi, \gamma_{1,2}\right) \leqslant k$. Further, at most two vertices, $\xi$ and $\gamma_{i, 1}$, are in $I\left(s_{i}\right)-\mathrm{A}$, where $s_{i}$ is the unique $\left[\gamma_{i-1,1}, \gamma_{i, 2}\right]$-path in $T, 2 \leqslant i \leqslant r$; since $T$ contains no $(A, k)$-solid path, $s_{i}$ cannot have length greater than $k$, so $d_{T}\left(\gamma_{i-1,1}, \gamma_{i, 2}\right) \leqslant k$ for $i \epsilon\{2, \ldots, r\}$. Since $T_{1}, \ldots, T_{r}$ satisfy the conditions of this lemma and have fewer than $q$ vertices each, in $T_{i}^{k}-A$ there is a Hamiltonian $\left[\gamma_{i, 2}, \gamma_{i, 1}\right]$-path $a_{i}$. Thus, $\xi,\left(a_{1}\right),\left(a_{2}\right), \ldots,\left(a_{r}\right)$ is a Hamiltonian $[\xi, \eta]$-path in $T^{k}-A$. 
Examples of trees $T$ and sets $A$ which satisfy the conditions of Lemma 3 are shown in figure 1, where the vertices in the sets $A$ are contained in dashed curves.

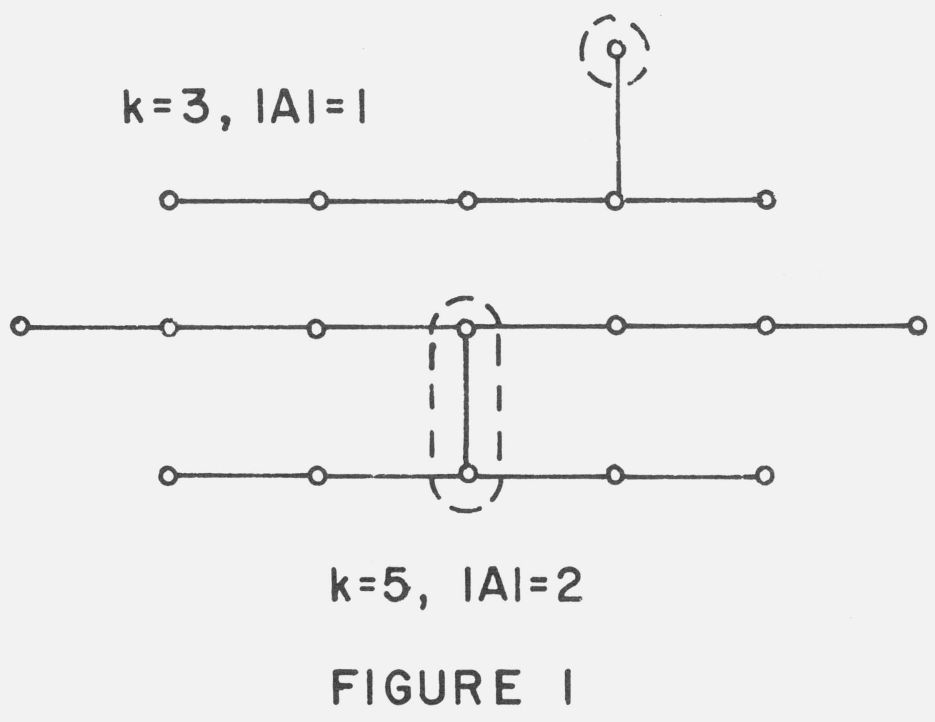

CoRollary 3a: Let $\mathrm{T}$ be a finite tree with at least 3 vertices, let $\mathrm{k}$ be an integer no less than 3 , and let $\mathrm{A} \subseteq \mathrm{V}(\mathrm{T})$ such that no path of $\mathrm{T}$ is $(\mathrm{A}, \mathrm{k})$-solid. Then, for each edge $\lambda \epsilon \mathrm{E}(\mathrm{T}-\mathrm{A}), \mathrm{T}^{\mathrm{k}}-\mathrm{A}$ contains a Hamiltonian circuit $\mathrm{c}$ with $\lambda \in \mathbf{E}(\mathrm{c})$.

Theorem 3: Let $\mathrm{T}$ be a finite tree, let $\mathrm{k}$ be an integer no less than 3, and let $\mathrm{A} \subseteq \mathrm{V}(\mathrm{T})$. Suppose $\mathrm{T}$ has no $(\mathrm{A}, \mathrm{k})$-solid paths. Then $\mathrm{T}^{\mathrm{k}}-\mathrm{A}$ is Hamiltonian connected.

Proof: The theorem is clearly true for a tree with 1 or 2 vertices, and it is vacuously true for a tree with no vertices. Suppose the theorem is true for every tree with fewer than $q$ vertices, and let $T$ be a tree with $q$ vertices. By Lemma 3, we need only show that, for any two distinct vertices which are not $A$-joined in $T, T^{k}-A$ contains a Hamiltonian path joining them. Let $\xi$ and $\eta$ be two vertices of $V(T)-A$ which are not $A$-joined. Let $a$ be the $[\xi, \eta]$-path in $T$ and let $\alpha \epsilon I(a)-A$. Let the components of $T-\alpha$ which contain vertices not in $A$ be $C_{1}, \ldots ., C_{r}$, with $\xi \in V\left(C_{1}\right)$ and $\eta \in V\left(C_{r}\right)$.

For $i \epsilon\{2,3, \ldots, r-1\}$, let $\rho_{i}$ be a vertex of $C_{i}-A$ which is $A$-joined to $\alpha$ in $T$. Let $\rho_{1}=\xi$. If there is a vertex of $C_{r}-A$ other than $\eta$ which is $A$-joined to $\alpha$, let $\rho_{r}$ be such a vertex, and if $\eta$ is the only vertex in $C_{r}-A$, let $\rho_{r}=\eta$. Otherwise, let $\rho_{r}$ be a vertex of $C_{r}-A$ which is $A$-joined to $\eta$. For $i \epsilon\{2,3, \ldots, r-1\}$, let $\delta_{i}$ be a vertex of $C_{i}-A$ other than $\rho_{i}$ which is $A$-joined to $\rho_{i}$ (if no such vertex exists, let $\delta_{i}=\rho_{i}$ ). If there is a vertex of $C_{1}-A$ other than $\xi$ which is $A$-joined to $\alpha$ in $T$, let $\delta_{1}$ be such a vertex, and if $\xi$ is the only vertex in $C_{1}-A$, let $\delta_{1}=\xi$. Otherwise, let $\delta_{1}$ be a vertex which is $A$-joined to $\xi$. Let $\delta_{r}=\eta$. By these choices, $\xi$ is the only vertex not in $A$ which can be on the path of $T$ joining $\delta_{1}$ and $\alpha$, and $\eta$ is the only vertex not in $A$ which can be on the path of $T$ joining $\alpha$ and $\rho_{r}$.

Each component $C_{i}$ is a tree with fewer than $q$ vertices. Further, since $T$ contains no $(A, k)$ solid paths, $C_{i}$ contains no $(A, k)$-solid paths, for each $i$. Thus the theorem holds for each tree $C_{i}$, and $C_{i}^{k}-A$ contains a Hamiltonian $\left[\rho_{i}, \delta_{i}\right]$-path $p_{i}$.

We note that in a tree $S$ with no $(A, k)$-solid paths, if $p$ is a path for which $\{F(p), L(p)\} \subseteq$ $V(S)-A$ and $|I(p)-A| \leqslant 2$, then $l(p) \leqslant k$. Hence, $d_{T}\left(\delta_{1}, \rho_{2}\right) \leqslant k$ since at most $\xi$ and $\alpha$ of $V(T)-A$ can be on the $\left[\delta_{1}, \rho_{2}\right]$-path of $T$. Also, $d_{T}\left(\delta_{i}, \rho_{i+1}\right) \leqslant k$ whenever $i \epsilon\{2,3, \ldots, r-2\}$, since at most $\rho_{i}$ and $\alpha$ of $V(T)-A$ can be on the $\left[\delta_{i}, \rho_{i+1}\right]$-path in $T$. Further, $d_{T}\left(\delta_{r-1}, \alpha\right) \leqslant k$ since $\rho_{r-1}$ is the only vertex not in $A$ which can be on the $\left[\delta_{r-1}, \alpha\right]$-path of $T$, and $d_{T}\left(\alpha, \rho_{r}\right) \leqslant k$ since $\eta$ is the only vertex not in $A$ which can be on the $\left[\alpha, \rho_{r}\right]$-path in $T$. Therefore, $\left(p_{1}\right),\left(p_{2}\right), \ldots,\left(p_{r-1}\right), \alpha,\left(p_{r}\right)$ is a Hamiltonian $[\xi, \eta]$-path in $T^{k}-A$. 
Corollary 3A: Let $\mathrm{G}$ be a connected finite graph, iet $\mathrm{k}$ be an integer no less than 3, and let $\mathrm{A} \subseteq \mathrm{V}(\mathrm{G})$ with $|\mathrm{A}| \leqslant \mathrm{k}-3$. Then $\mathrm{G}^{k}-\mathrm{A}$ is Hamiltonian connected.

Proof: Let $T$ be a spanning tree in $G$. Since $|A| \leqslant k-3, T$ contains no $(A, k)$-solid paths by Lemma 1. Therefore, $T^{k}-A$ is Hamiltonian connected by Theorem 3. But $T^{k}-A$ is a spanning subgraph of $G^{k}-A$; thus $G^{k}-A$ is Hamiltonian connected.

Corollary 3A proves Conjecture 1 for the case of $\kappa(G)=1$. We can improve this result slightly in the direction of Conjecture 1 as follows:

CoRollaRY 3B: Let $\mathrm{G}$ be a connected finite graph and let $\mathrm{k}$ be an integer no less than 3. Let $\mathrm{A} \subseteq \mathrm{V}(\mathrm{G})$ with $|\mathrm{A}| \leqslant \mathrm{k}+\kappa(\mathrm{G})-4$. Then $\mathrm{G}^{\mathrm{k}}-\mathrm{A}$ is Hamiltonian connected.

Proof: $A$ can be expressed as the union of disjoint sets $B$ and $C$ such that $|B| \leqslant k-3$ and $|C| \leqslant \kappa(G)-1$. From the definition of $\kappa(G), G-C$ is connected; thus $(G-C)^{k}-B$ is Hamiltonian connected by Corollary $3 \mathrm{~A}$. But $(G-C)^{k}$ is a spanning subgraph of $G^{k}-C$ and $B \cup C=A$; hence $(G-C)^{k}-B$ is a spanning subgraph of $G^{k}-A$, and $G^{k}-A$ is Hamiltonian connected.

LeMmA 4: Let $\mathrm{k}$ be an integer no less than 2, let $\mathrm{G}$ be a graph, and let $\mathrm{A} \subseteq \mathrm{V}(\mathrm{G})$ such that $|\mathrm{A}| \leqslant \mathrm{k}-2$. Let $\mathrm{p}$ be an $(\mathrm{A}, \mathrm{k})$-solid path in $\mathrm{G}$. Then $\mathrm{A} \subseteq \mathrm{I}(\mathrm{p})$.

Proof: By definition, $l(p)=|I(p)|+1$ and $|I(p)-A| \leqslant 2$. Therefore, $k<l(p)=|I(p)|+1$ $=|I(p) \cap A|+|I(p)-A|+1 \leqslant|I(p) \cap A|+3$, or $k-2 \leqslant|I(p) \cap A|$. But $|A| \leqslant k-2$. Thus, $A \subseteq I(p)$.

In a paper published in 1960, Sekanina [18] proved

Theorem (Sekanina's Theorem): If $\mathrm{G}$ is a connected finite graph, then $\mathrm{G}^{3}$ is Hamiltonian connected.

This theorem was proved again by Karaganis in a paper [15] published in 1968. Using this result, Chartrand and Kapoor [2] proved

THEOREM A: If $\mathrm{G}$ is a connected finite graph with at least 4 vertices, then $\mathrm{G}^{3}$ is 1-Hamiltonian.

A proof by construction for the next theorem was recently published in [1]. However, the following proof is belived to have points of sufficient interest to warrant its publication. Recall that if $p$ is a path, then $/ p /$ is the graph whose edges and vertices are precisely those of $p$.

Theorem 4: Let $\mathrm{k}$ be an integer no less than 3, and let $\mathrm{G}$ be a connected finite graph with at least $\mathrm{k}+1$ vertices. Then $\mathrm{G}^{\mathrm{k}}$ is $\mathrm{r}$-Hamiltonian for every integer $\mathrm{r}$ in $\{0,1, \ldots, \mathrm{k}-2\}$.

Proof: Let $A \subseteq V(G)$ with $|A| \leqslant k-2$. If $|A| \leqslant 1$, then $G^{3}-A$ contains a Hamiltonian circuit by Theorem A. Since $G^{3}-A$ is a spanning subgraph of $G^{k}-A, G^{k}-A$ is Hamiltonian.

Suppose $|A| \geqslant 2$. Since $G$ is connected, we may choose a spanning tree $T$ of $G$. If $T$ has no $(A, k)$-solid paths, then $T^{k}-A$ is Hamiltonian connected by Theorem 3 , and so it contains a Hamiltonian circuit. Since $T^{k}-A$ is a spanning subgraph of $G^{k}-A, G^{k}-A$ is Hamiltonian.

Now we assume that $T$ contains an $(A, k)$-solid path $a$; by Lemma $4, A \subseteq I(a)$. Since $|A| \geqslant 2$, there is an edge $\lambda=\left(\xi_{1}, \xi_{2}\right)$ in $E(a)$ such that each component of $|a|-\lambda$ contains one or more vertices of $A$. Let $T_{i}$ be the component of $T-\lambda$ which contains $\xi_{i}, i \epsilon\{1,2\}$. For $i \epsilon\{1,2\}$, if $\xi_{i} \notin A$, we let $\rho_{i}=\xi_{i}$, and if $\xi_{i} \in A$, we let $\rho_{i}$ be a vertex of $T_{i}$ which is not in $A$ and which is $A$-joined to $\xi_{i}$ (such a vertex exists because the ends of $a$ are not in $A$ ). Let $\delta_{i}$ be a vertex of $V\left(T_{i}\right)-A$ different from $\rho_{i}$ which is $A$-joined to $\rho_{i}$ (if no such vertex exists, let $\delta_{i}=\rho_{i}$ ). Since both of $T_{1}$ and $T_{2}$ contain vertices of $A,\left|V\left(T_{i}\right) \cap A\right|<k-2$ for each $i$; hence $T_{i}$ satisfies the conditions of Lemma 1 and contains no $(A, k)$-solid arc. Then applying Lemma 3 to $T_{i}, i \epsilon\{1,2\}$, let $a_{i}$ be a Hamiltonian path in $T_{i}^{k}-A$ from $\rho_{i}$ to $\delta_{i}$ (if $\rho_{i}=\delta_{i}$, let $a_{i}=\rho_{i}$ ). Since $\rho_{1}$ is the only vertex of $V(T)-A$ which can lie on the $\left[\delta_{1}, \rho_{2}\right]$-path of $T, d_{T}\left(\delta_{1}, \rho_{2}\right) \leqslant k$ by Lemma 2 . Similarly, $d_{T}\left(\delta_{2}, \rho_{1}\right) \leqslant k$. Hence $\left(a_{1}\right),\left(a_{2}\right)$, $\rho_{1}$ is a Hamiltonian circuit in $T^{k}-A$, and it is also a Hamiltonian circuit in $G^{k}-A$.

The following example shows that Theorem 4 cannot be strengthened to the level of Theorem 3 , and Corollary $3 \mathrm{~A}$ is best possible in the usual sense. In the tree $T$ shown in figure $2 \mathrm{~A}$, the five vertices surrounded by the dashed curve are the vertices in $A$, and $k=7$. $T^{7}-A$ is shown in figure 2B. In $T, \alpha$ and $\beta$ are $A$-joined, but it is easily shown that in $T^{7}-A$, there is no Hamiltonian $[\alpha, \beta]$ path ( $\gamma$ would have to be both the successor of $\alpha$ and the predecessor of $\beta$ in any such path). 
The following corollary provides a slight weakening of the conditions of Theorem 4, with a corresponding weakening of the conclusion:
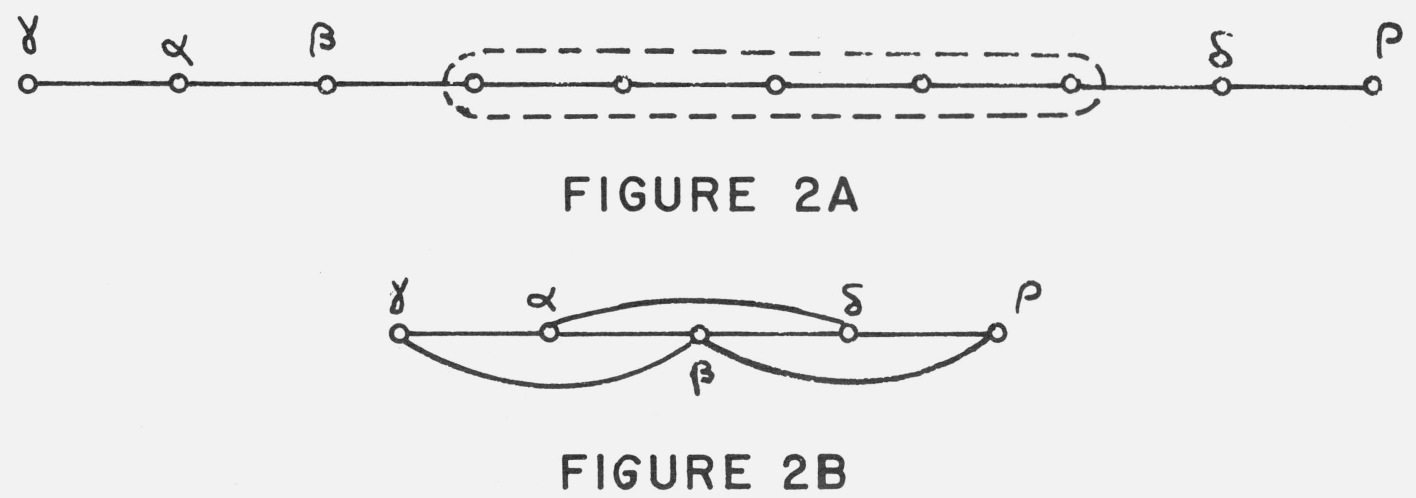

COROLlaRY 4A: Let $\mathrm{G}$ be a connected finite graph with at least 3 vertices, let $\mathrm{k}$ be an integer no less than 3 , and let $\mathrm{A}$ be a subset of $\mathrm{V}(\mathrm{G})$ such that $|\mathrm{A}| \leqslant \min (\mathrm{k}-1,|\mathrm{~V}(\mathrm{G})|-2)$. Then $\mathrm{G}^{\mathrm{k}}-\mathrm{A}$ contains a Hamiltonian path.

Proof: We simply remove all but one of the vertices of $A$ from $G^{k}$. By Theorem 4 , the resulting graph contains a Hamiltonian circuit. Removing one more vertex converts the circuit into a Hamiltonian path in the resulting graph, which is $G^{k}-A$.

While we have not proved Conjecture 2, Theorem 4 allows us to come a little closer to the statement of the conjecture, as the following corollary shows:

Corollary 4B: Let $\mathrm{k}$ be an integer no less than 3 and let $\mathrm{G}$ be a connected finite graph with at least $\mathrm{k}+\kappa(\mathrm{G})$ vertices. Then $\mathrm{G}^{\mathrm{k}}$ is $\mathrm{r}$-Hamiltonian for every $\mathrm{r}$ in $\{0,1, \ldots, \mathrm{k}+\kappa(\mathrm{G})-3\}$.

The proof of this corollary is similar to the proof of Corollary $3 \mathrm{~B}$.

Conjecture 2 might be extended to include the following:

Conjecture 3: Let $\mathrm{G}$ be a graph with $\kappa(\mathrm{G}) \geqslant 2$ and with at least $2 \kappa(\mathrm{G})+1$ vertices. Then $\mathrm{G}^{2}$ is $(2 \kappa(\mathrm{G})-2)$-Hamiltonian.

I suspect that Conjecture 3 does not hold (although it is valid if $G$ is a circuitoid). However, the following weaker theorem was recently proved [3, 14]:

THEOREM: Let $\mathrm{G}$ be a graph with $\kappa(\mathrm{G}) \geqslant 2$ and with at least 4 vertices. Then $\mathrm{G}^{2}$ is 1 -Hamiltonian.

\section{Squares of Graphs}

The set of all vertices of $G$ having degree $k$ is denoted by $V_{k}(G)$. A caterpillar is a tree $T$ such that $T-V_{1}(T)$ is a pathoid or the empty graph. A 2 -factor of a graph $G$ is a subgraph $H$ of $G$ such that $V(H)=V(G)$ and every vertex in $H$ has degree 2 .

In contrast to the situation for higher powers of graphs, we do not yet know which graphs have Hamiltonian squares. Neuman [16] has characterized those trees for which a Hamiltonian path joining two specified vertices will exist in the square of the trees. An easy consequence of his characterization is the following result:

THEOREM 5: The square of a tree is Hamiltonian if and only if the tree is a caterpillar with at least 3 vertices. ${ }^{2}$

H. Fleischner $[6,7,8,9]$ has proved that the square of a block is Hamiltonian, and he has characterized those cubic graphs with Hamiltonian squares [5, 10]. A few other more special results are known. The remainder of this paper contains two further theorems connected with the problem of determining which graphs have Hamiltonian squares.

${ }^{2}$ Neuman's result can also easily be used to show that the square of a tree is Hamiltonian connected if and only if the tree is a caterpillar with at most one vertex of degree greater than one. 
If the square of a graph contains a Hamiltonian circuit, it certainly contains a 2 -factor. Thus, it is reasonable to consider the question of which squares of graphs contain 2 -factors $\left(S\left(K_{1,3}\right)\right.$ is an example of a graph whose square does not contain a 2 -factor). In this connection, the following theorem is of interest. Given a path $p$, the internal vertices of $p$ are the vertices in $I(p)$. We denote the degree in $G$ of a vertex $\xi$ by $v_{G}(\xi)$.

Theorem 6: Let $\mathrm{G}$ be a graph with minimum degree at least 2. Then $\mathrm{G}^{2}$ contains a 2-factor.

Proof: By Theorem 5, it is sufficient to find a spanning forest in $G$ in which each tree is a caterpillar with at least 3 vertices. We note first that since $G$ has minimum degree at least 2 , each component of $G$ contains at least 3 vertices, and a longest path in $G$ must have length at least 2 .

Choose a longest path $a_{1}$ in $G$. Having chosen paths $a_{1}, \ldots, a_{k}$ in $G$, if $H_{k}=G-\bigcup_{i=1}^{k} V\left(a_{i}\right)$ is not empty, choose a longest path $a_{k+1}$ in $H_{k}$. Note that the end vertices of $a_{k+1}$ can be adjacent only to vertices in $\cup_{i=1}^{k} I\left(a_{i}\right) \cup V\left(a_{k+1}\right)$. Eventually, since $G$ is finite, we find we have chosen paths $a_{1}, \ldots, a_{r}$ such that $H_{r}$ is the empty graph. Now for each end vertex $\mu$ of each path of length 0 or 1 , choose an internal vertex of a longer path to which $\mu$ is adjacent through an edge $\lambda_{\mu}$ (such an internal vertex must exist since $v_{G}(\xi) \geqslant 2$ for all $\left.\xi \epsilon V(G)\right)$. We form a spanning forest whose trees are caterpillars by deleting the edges from those pathoids in $\left\{\left|a_{1} /, \ldots,\right| a_{r} /\right\}$ which have length 1 and adding the edges $\lambda_{\mu}$ to form the caterpillars. Since each of these caterpillars includes the vertices of a path with an internal vertex, each caterpillar has at least three vertices.

An Euler graph is a graph in which every vertex has even degree. In a path $p=(r), \xi,(s)$, the neighbors of the vertex $\xi$ are the vertices $L(r)$ and $F(s)$. Given a path $p=\xi_{1}, \xi_{2}, \ldots, \xi_{r-1}, \xi_{r}$, we let $p^{-1}$ denote the path $\xi_{r}, \xi_{r-1}, \ldots, \xi_{2}, \xi_{1}$. We call a path $x$ a section of a path $p$ iff there exist paths $y$ and $z$ such that $p=(y),(x),(z)$ ( $y$ or $z$ may be empty). Recall that all walks in this paper are denoted by sequences of vertices.

The next theorem is a beginning for the study of the question of which Euler graphs have Hamiltonian squares. Fleischner's proof that the square of a block is Hamiltonian involves finding a Hamiltonian circuit in the square of an Euler graph (see especially [7]); thus, a thorough understanding of Euler graphs with Hamiltonian squares would be quite desirable. The following Theorem 7, whose statement is quite long, can be summarized as follows: "If $G$ is an Euler graph with at least 3 vertices such that $G-V_{2}(G)$ is a forest, then $G^{2}$ is Hamiltonian. Further, $G^{2}$ contains a Hamiltonian circuit which includes many of the edges of $G$."

THEOREM 7: Let $G$ be a connected Euler graph with at least 3 vertices such that $G-V_{2}(G)$ is a forest with trees $\mathrm{T}_{1}, \ldots, \mathrm{T}_{\mathrm{r}}, \mathrm{r} \geqslant 0$. Let $\mathrm{e}$ be an Euler trail in $\mathrm{G}$. In each tree $\mathrm{T}_{\mathrm{i}}, \mathrm{i} \epsilon\{1,2, \ldots, \mathrm{r}\}$, choose one vertex $\eta_{\mathrm{i}}$ and call it the root of $\mathrm{T}_{\mathrm{i}}$. Then $\mathrm{G}^{2}$ contains a Hamiltonian circuit $\mathrm{h}$ such that:

(1) $\mathrm{h}$ is a subsequence of $\mathrm{e}$, where both $\mathrm{h}$ and $\mathrm{e}$ are denoted by a sequence of vertices;

(2) the root $\eta_{\mathrm{i}}$ of each tree $\mathrm{T}_{\mathrm{i}}$ is adjacent in $\mathrm{G}$ to its two neighbors in $\mathrm{h}$; and

(3) for each path $\mathrm{s}$ in $\mathrm{G}$ such that $\mathrm{V}(\mathrm{s}) \subseteq \mathrm{V}_{2}(\mathrm{G})$, either $\mathrm{s}$ or $\mathrm{s}^{-1}$ is a section of $\mathrm{h}$.

Proof: The theorem is trivial if $G$ is a circuitoid. Thus we may assume $r \geqslant 1$. Define a function $\Psi$, with domain $V(G)-\left(V_{2}(G) \cup\left\{\eta_{1}, \eta_{2}, \ldots, \eta_{r}\right\}\right)$, as follows: for $\xi \neq \eta_{i}$ in $V\left(T_{i}\right)$, let $\Psi(\xi)$ be the second vertex on the unique $\left[\xi, \eta_{i}\right]$-path in $T_{i}$. In $e$, mark each vertex of $V_{2}(G)$, and mark one occurrence of each of $\eta_{1}, \ldots, \eta_{r}$. Also for each vertex $\xi \in V(G)-\left(V_{2}(G) \cup\left\{\eta_{1}, \ldots, \eta_{r}\right\}\right)$, mark precisely that occurrence of $\xi$ in $e$ for which $\Psi(\xi)$ is a neighbor of $\xi$ at that location in $e$. Form $h$ from $e$ by deleting all unmarked vertices in $e$. This choice of $h$ satisfies condition (1) of the theorem.

Since we left in $h$ one occurrence of each of the distinct vertices in $e$, and since $V(e)=V(G)$, $V(h)=V(G)$. Note that the unmarked vertices of $e$ are all in the forest $G-V_{2}(G)$. Two vertices of $G-V_{2}(G)$ which are successive in $e$ and are adjacent in that subgraph are in the same tree $T_{i}$. Hence one is the second vertex in the path joining the other to $\eta_{i}$, so that one of them is necessarily marked at that part of $e$. Thus no two successive vertices in $e$ are both unmarked, and for every section $\omega, \chi$ of $h$, there is at most one vertex between $\omega$ and $\chi$ in $e$ at the corresponding location in $e$. Since $e$ is a trail in $G, d_{G}(\omega, \chi) \leqslant 2$. Thus, $h$ is a Hamiltonian circuit in $G^{2}$. 
For each $i \epsilon\{1,2, \ldots, r\}$, since $\eta_{i}$ is $\Psi(\xi)$ for every vertex $\xi$ adjacent to $\eta_{i}$ in $T_{i}$, and since no vertex of degree 2 in $G$ is deleted in obtaining $h$ from $e$, no neighbors of $\eta_{i}$ in $e$ are deleted when forming $h$. Thus, in the one occurrence of $\eta_{i}$ in $h$, both neighbors of $\eta_{i}$ in $h$ are neighbors of $\eta_{i}$ in $e$ and hence are adjacent to $\eta_{i}$ in $G$. Finally, for each path $s$ in $G$ such that $V(s) \subseteq V_{2}(G)$, either $s$ or $s^{-1}$ is a section of $e$. Since no vertices of degree 2 in $G$ are deleted in forming $h, s$ or $s^{-1}$ remains a section of $h$. Thus $h$ satisfies conditions (2) and (3) of the theorem.

For any $\xi \in V(G)$, if $\xi$ and $\eta_{i}$ are adjacent in $G$ they must be neighbors at some occurrence of $\eta_{i}$ in $e$. Then at that occurrence of $\eta_{i}, \eta_{i}$ can be marked. Thus, for each root $\eta_{i}$, we may select one vertex adjacent to $\eta_{i}$ in $G$ which will be a neighbor of $\eta_{i}$ in $h$.

Let $G$ be a graph whose square is Hamiltonian, let $h$ be a Hamiltonian circuit in $G$, and let $\lambda$ be an edge of $G$ in $h$. Let $\xi$ be one of the ends of $\lambda$. Further, let $H_{1}$ and $H_{2}$ be disjoint caterpillars disjoint from $G$, and let $\eta_{i}$ be a vertex of degree no more than 1 in $H_{i}-V_{1}\left(H_{i}\right)$, for $i \epsilon\{1,2\}$ (if $H_{i}$ has only two vertices, let $\eta_{i}$ be one of them). Form a graph $M$ from $H_{1}$ and $G$ by identifying $\eta_{1}$ and $\xi$. Then $M^{2}$ is Hamiltonian as indicated by figure 3 , where the dashed line indicates the sequence inserted between the ends of $\lambda$ in $h$, (in this and following figures, a wavy line is used to denote $h$ ). V ariations in the length of $H_{1}-V_{1}\left(H_{1}\right)$ (particularly variations to odd lengths) and changes in the number of vertices of degree 1 in $H_{1}$ can be dealt with in an obvious manner. Note that one edge of $H_{1}$ is included in the Hamiltonian circuit of $M^{2}$.

Now let $H_{1}$ and $H_{2}$ be as before, but suppose $G^{2}$ contains a Hamiltonian circuit $h$ which includes two edges $\lambda$ and $\mu$ of $G$, both incident with the same vertex $\xi$ of $G$. In this case, let $N$ be formed from $H_{1}, H_{2}$, and $G$ by identifying $\eta_{1}, \eta_{2}$, and $\xi$. Then $N^{2}$ contains a Hamiltonian circuit as indicated by figure 4 , where the dashed lines indicate the sequence which is to replace $\xi$ in $h$. Variations in $H_{1}$ and $H_{2}$ are again easily dealt with. Note that an edge of each of $H_{1}$ and $H_{2}$ is in the Hamiltonian circuit of $N^{2}$.

Now let $G$ and $H$ be disjoint graphs such that $G^{2}$ and $H^{2}$ are both Hamiltonian. Let $\lambda$ be an edge of $G$ incident with a vertex $\xi$ and in a Hamiltonian circuit $g$ of $G^{2}$, and let $\mu$ be an edge of $H$ incident with a vertex $\eta$ and in a Hamiltonian circuit $h$ of $H^{2}$. Form a graph $P$ from $G$ and $H$ by identifying $\xi$ and $\eta$. Then $P^{2}$ contains a Hamiltonian circuit as indicated by the wavy and dashed curves in figure 5 .

Theorem 7 describes many edges of $G$ which are included in a Hamiltonian circuit of $G^{2}$, where $G$ is an Euler graph in which $G-V_{2}(G)$ is a forest. In view of the techniques described in the preceding three paragraphs, Theorem 7 can thus be used to show many other graphs have Hamiltonian squares.

An Euler graph $G$ whose square is not Hamiltonian is shown in figure 6 . It is easy to see that any Hamiltonian circuitoid in $G^{2}$ must contain edges joining a vertex other than a cut vertex of $G$ of each of the small triangles $T_{1}, T_{2}, T_{3}, T_{4}$, and $T_{5}$ to one of $\alpha$ or $\beta$. Thus one of $\alpha$ or $\beta$ must meet three edges

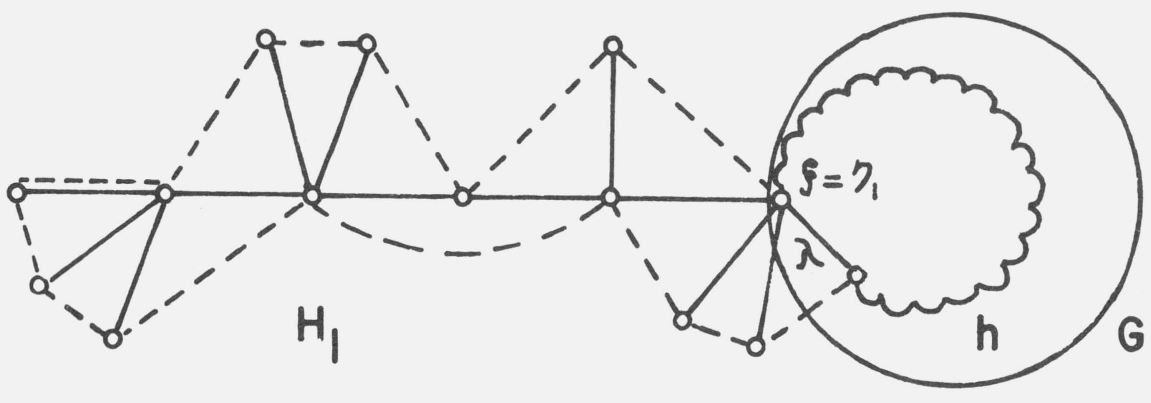

FIGURE 3 


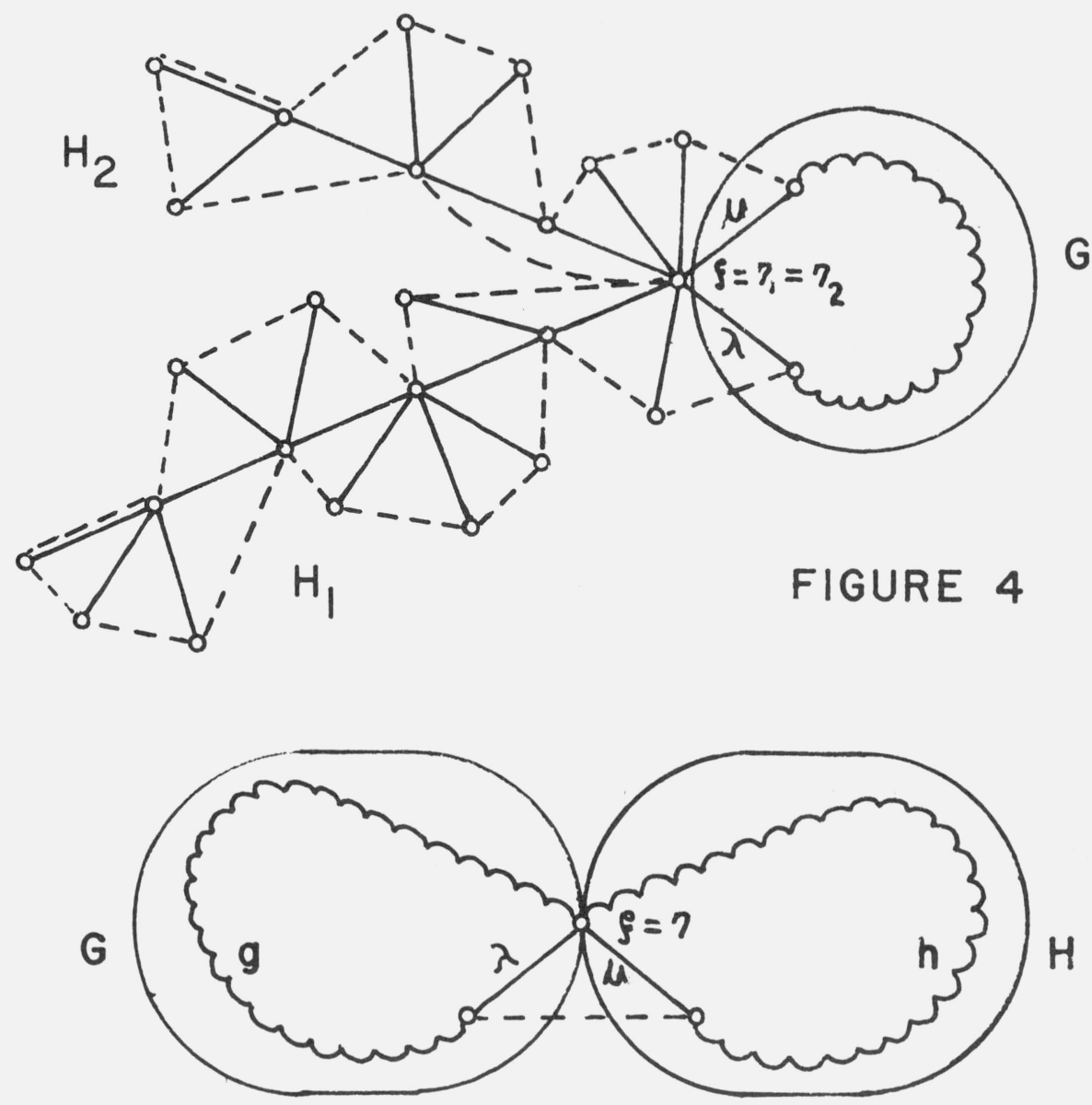

FIGURE 5

of the Hamiltonian circuitoid, which is impossible. I suspect that the graph of figure 6 is the smallest Euler graph whose square is not Hamiltonian (size being measured in number of vertices or number of edges).

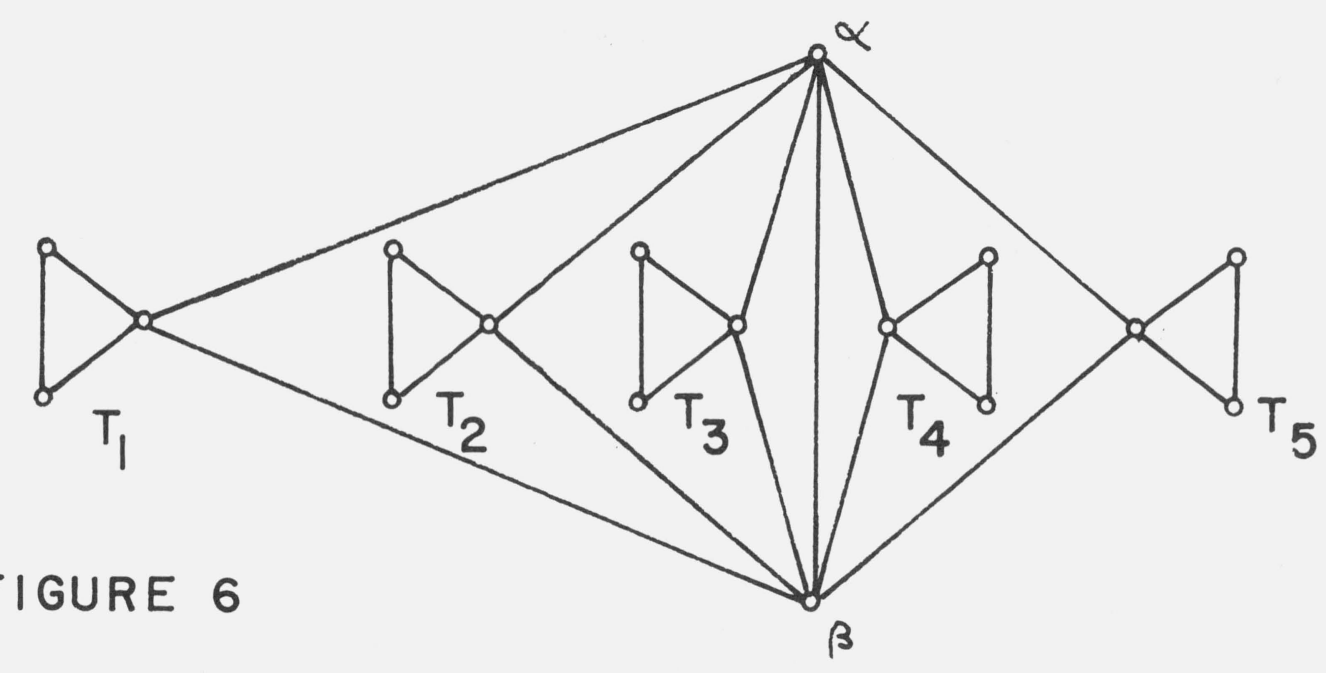


ADDED IN PROOF: Since this paper was submitted, references [6] and [7] have been combined into Fleischner, H., On spanning subgraphs of a connected bridgeless graph and their application to $D T$-graphs, J. Combinatorial Theory (to appear), and references [8] and [9] were combined into Fleischner, H., The square of every two-connected graph is Hamiltonian, J. Combinatorial Theory (to appear). Further, references [3] and [14] were combined into Chartrand, G., Hobbs, A. M., Jung, H. A., Kapoor, S. F., and Nash-Williams, C. St. J. A., The square of a block is Hamiltonian connected, J. Combinatorial Theory (to appear). It should be mentioned that the term " $\xi$-section" is similar to the term " $J$-component" of Tutte, W. T., The Connectivity of Graphs (Toronto Univ. Press, Toronto, 1967), and that many of the other terms in this paper not found in [11] were derived from the definitions in Nash-Williams, C. St. J. A., Graph-Theoretic Definitions (unpublished).

My thanks go to Professors W. T. Tutte and C. St. J. A. Nash-Williams for their many useful suggestions. In particular, I wish to thank Professor Nash-Williams for suggesting the present form of the proof of Theorem 7 .

\section{References}

[1] Bhat, V. N., and Kapoor, S. F., The powers of a connected graph are highly Hamiltonian, J. Res. Nat. Bur. Stand. (U.S.), 75B (Math. Sci.) Nos. 1 \& 2, 63-66 (Jan.-June 1971).

[2] Chartrand, G., and Kapoor, S. F., The cube of every connected graph is 1-Hamiltonian, J. Res. Nat. Bur. Stand. (U.S.), 73B (Math. Sci.) No. 1, 47-48 (Jan.-Mar. 1969).

[3] Chartrand, G., and Kapoor, S. F., The square of every 2-connected graph is 1-Hamiltonian (to appear).

[4] Chartrand, G., Kapoor, S. F., and Lick, D. R., n-Hamiltonian graphs, J. Combinatorial Theory 9, 308-312 (1970).

[5] Fleischner H., Über Hamiltonsche Linien im Quadrat kubishcer und pseudokubischer Graphen (to appear).

[6] Fleischner, H., On a certain spanning subgraph of a connected bridgeless graph (to appear).

[7] Fleischner, H., The total graph of a block is Hamiltonian (to appear).

[8] Fleischner, H., On line-critical blocks (to appear).

[9] Fleischner, H., The square of every non-separable graph is Hamiltonian (to appear).

[10] Fleischner, H., Zum Quadrat kubischer Graphen (to appear).

[11] Harary, F., Graph Theory (Addison-Wesley Publishing Co., Reading, Mass., 1969).

[12] Hobbs, A. M., Some Hamiltonian results in the square of a graph, Notices Amer. Math. Soc. 18, 397 (1971).

[13] Hobbs, A. M., A catalog of minimal blocks (to appear).

[14] Hobbs, A. M., and Nash-Williams, C. St. J. A., The square of a block is Hamiltonian connected (to appear).

[15] Karaganis, J. J., On the cube of a graph, Canad. Math. Bull. 11, 295-296 (1968).

[16] Neuman, F., On a certain ordering of the vertices of a tree, Casopis Pěst. Mat. 89, 323-339 (1964).

[17] Ore, O., Hamiltonian connected graphs, J. Math. Pures Appl. 42, 21-27 (1963).

[18] Sekanina, M., On an ordering of the set of vertices of a connected graph, Publ. Fac. Sci. Univ. Brno, Tchecoslovaquie, No. 412, 137-141 (1960).

(Paper 77B1 \& 2-374) 